\title{
The Effect of Atmospheric Plasma on the Solubility and Dispersibility of Powdered Whey Protein Isolate
}

\author{
Heidi Lightfoot
}

Clinical Research Physician, Basingstoke and North Hampshire Hospital, England Email: heidi.lightfoot@gmail.com 


\begin{abstract}
:
Functional properties of protein macromolecules such as protein solubility are of particular interest to the food and nutrition industries as they have significant implications on other useful properties and characteristics for the development of nutritional and food supplements. Consequently, proteins with specific and consistent functional characteristics are in high demand as essential ingredients in formulated food or in pharmaceutical and industrial mixtures. Proteins need to be highly soluble so that their functional properties can be effectively exploited, therefore methods to improve the solubility of protein powders are currently being developed.

It has been hypothesized that atmospheric plasma treatment has an effect on protein solubility and dispersibility. This theory has not been yet explored with whey protein isolate elsewhere; this study is the first to explore the impact of plasma based treatment. The effect of atmospheric plasma treatment on the solubility and dispersibility of dry protein powder has been studied. Each variable was examined using both a pristine sample of whey protein isolate and a sample of whey protein isolate from the same product batch that had been exposed to atmospheric plasma (following ISO 8156 and ISO/TS 17758 protocols). We demonstrate that plasma can successfully increase the solubility and dispersibility of whey protein powder.
\end{abstract}

\title{
Figures:
}


A.

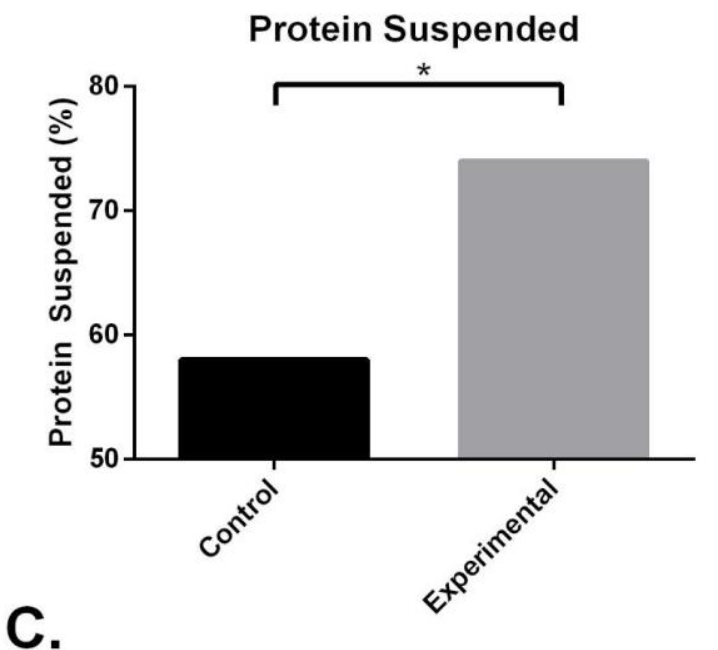

Protein $\mathrm{mg} / \mathrm{mL}$ Added Vs. Suspended

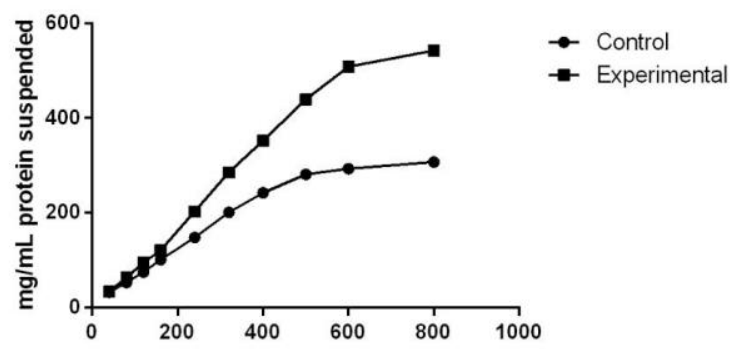

E.

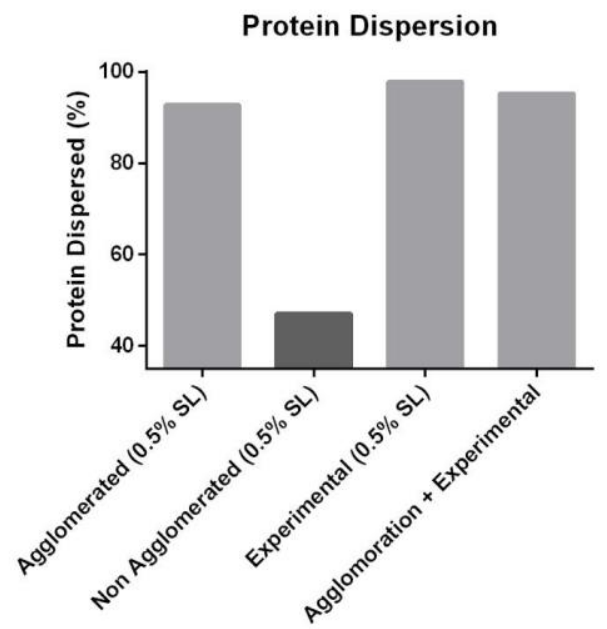

B.
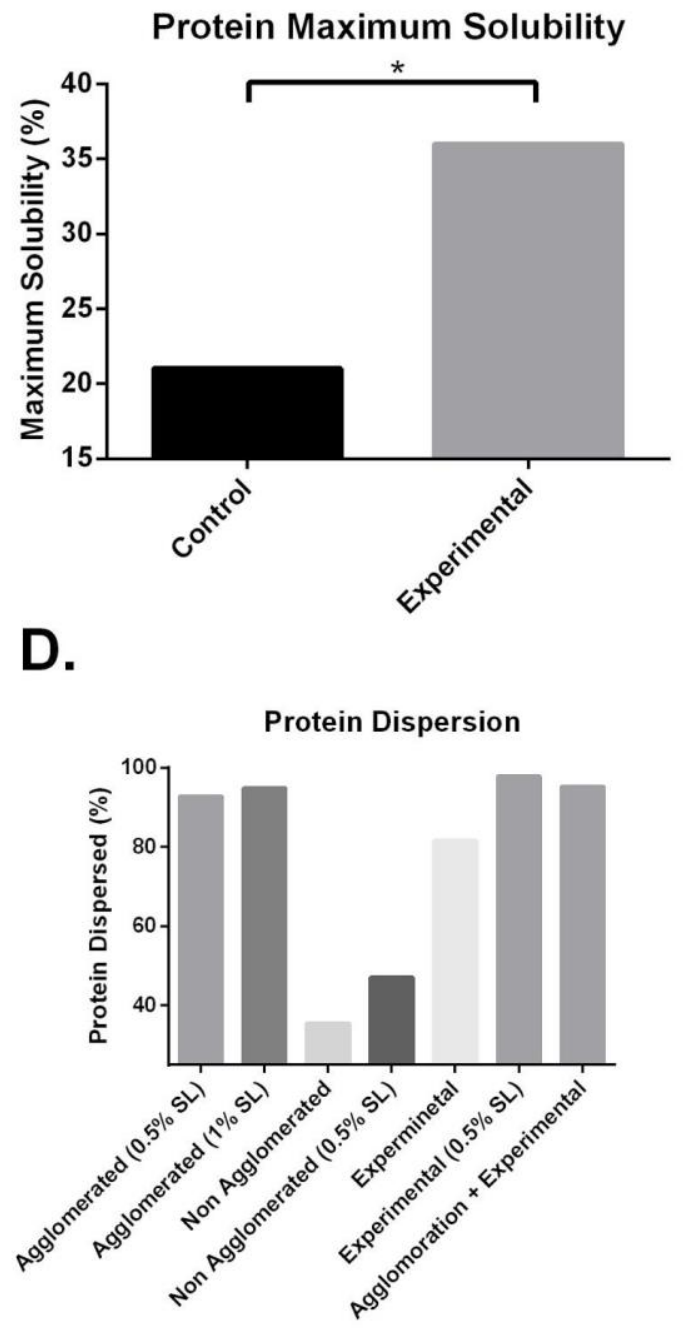


\section{Figure Legend:}

Figure A: Percent of protein suspended at a "typical" protein dose of a $30 \mathrm{~g}$ scoop in 8 ounces of water. Asterisk $\left(^{*}\right)$ indicates $27.58 \%$ increase in protein suspended with PSM treatment compared to untreated control.

Figure B: Percent of protein at maximum solubility (completely dissolved, no particulate or clumped to any degree). Asterisk $\left({ }^{*}\right)$ indicates $71.43 \%$ increase in maximum solubility with PSM treatment as compared to untreated control.

Figure C: Protein added to water $(\mathrm{mg} / \mathrm{mL})$ compared to protein suspended with PSM treatment $(\mathrm{mg} / \mathrm{mL})$ compared to untreated control.

Figure D: Comparison of protein dispersion of agglomerated protein $(0.5 \%$ and $1 \%$ Soy Lecithin), non agglomerated ( $0.0 \%$ Soy Lecithin and $0.5 \%$ Soy Lecithin), experimental $(0.0 \%$ Soy Lecithin and $0.5 \%$ Soy Lecithin) as well as experimental processing on an already agglomerated protein.

Figure E: Comparing protein dispersion of agglomerated protein ( $0.5 \%$ Soy Lecithin), non agglomerated ( $0.5 \%$ Soy Lecithin), experimental ( $0.5 \%$ Soy Lecithin) as well as experimental processing on an already agglomerated protein.

\section{Introduction:}

The production of food and nutritional supplements depends on a variety of functional features of powder based substances. Dry protein powder is popular for this purpose because it has many useful functional characteristics. The two most advantageous for use in the production of consumer products are solubility and dispersibility. Solubility of a protein refers to the capacity completely to dissolve in a liquid ${ }^{1}$; dispersibility is the tendency or readiness to disperse in water. ${ }^{2}$ It is the solubility characteristics of a protein that determine its subsequent properties of use in the food and nutrition industries. ${ }^{3,4}$

Previous investigations have focused on the effects of $\mathrm{pH}$ and temperature on the ability of a protein to dissolve in a liquid 5 ; this investigation sought to explore the impact of plasma-surface modification (PSM) on the solubility, dispersibility and suspension properties of dry protein powder. Currently, the process of agglomeration is the leading method for improving the solubility and dispersibility of powder based substances, 
including dry powder whey protein isolate, but PSM is a developing viable alternative method. This study compares PSM treated dry protein powder with agglomerated dry protein powder and non-agglomerated dry powder as methods for improving solubility and dispersibility.

Plasma treatments are commonly referred to as plasma-surface modification (PSM). They are widely accepted as effective surface-altering processes that can result in several changes of the original state. The exact effects depend on the treatment conditions and include surface-etching, cross-linking and alteration of surface functions. Plasma is a gas that is charged with electrons in both the negative and positive state. It is highly conductive and has high internal energy. ${ }^{6}$ The actual plasma treatment procedure takes place in a vacuum chamber with easily controllable pressure and temperature settings. ${ }^{7}$ This study is the first to examine atmospheric plasma as a means to directly induce changes in the structure of organic protein powders and thereby alter their solubility and dispersibility. Here we report an enhancement of both characteristics in whey protein isolate following PSM treatment.

\section{Objective:}

The goal of this study is to investigate whether protein structural alterations by atmospheric plasma modify the dispersibility and solubility of dry powdered whey protein isolate.

\section{Results, \& Discussion:}

This investigation focused on assessing the impact of plasma-surface modification on dry powdered whey protein isolate. A commercially available dry powder whey protein isolate was obtained as the source material for the study, which also served as the control for the experiment. A portion of the whey protein isolate from this batch was separated and exposed to atmospheric plasma via PSM. Proprietary PSM technology (Ingredient Optimization TM, Plasma Nutrition, USA) was used to effectively mediate the exposure of organic dry powder materials to plasma to enact multiple changes, including peptide level alterations and surface-level alterations. Following PSM treatment, solubility and dispersibility (ISO 8156ANS and ISO/TS 17758) analyses were conducted.

We first investigated the maximum percentage of dry powdered whey protein that could remain in suspension. The test aimed to mimic normal protein powder use, with $30 \mathrm{~g}$ of protein in 8 ounces of water. We found that PSM treated protein samples suspended $27.58 \%$ more protein compared to non-treated control (Figure 1). 
To assess the impact of PSM on dry protein powder solubility, we examined maximum solubility, defined as amount of product being completely dissolved, with no particulate of protein clumping to any degree. A total of $71.43 \%$ increase in maximum solubility with PSM treated protein powder was demonstrated compared to non-treated control (Figure 2). We also tested the relationship between protein volume and the amount of protein that remained in suspension, which was obtained by comparing the amount of protein added to water $(\mathrm{mg} / \mathrm{mL})$ to the amount of suspended protein $(\mathrm{mg} / \mathrm{mL})$. The experimental protein yielded higher values at every data point measured (Figure 3 ) as well as a peak $71.43 \%$ increase (Figure 2).

Our final investigation focused on the impact of PSM on dry protein powder dispersibility of six dry protein powder variants using ISO protocol 17758. Out of the total six, two samples were agglomerated dry protein powders with $0.5 \%$ and $1.0 \%$ Soy Lecithin, respectively. The first PSM sample was blended with $0.5 \%$ Soy Lecithin and the second blended with $1.0 \%$ Soy Lecithin. The final sample was agglomerated before plasma treatment (Figures 4 and 5). The dispersibility of PSM treated protein is at least statistically equal to agglomerated WPI, if not better. Furthermore, we demonstrate that exposing an already agglomerated protein to PSM does not reverse the initial benefits of agglomeration (Figures 4 and 5).

Overall, we show that both the solubility and dispersibility of a dry powdered whey protein isolate is increased by PSM treatment. The obtained dispersion levels were the same as those achieved via agglomeration (the alternative technology to increase dispersion characteristics of powders currently more widely used). This may suggest PSM as a more cost effective alternative for increasing the dispersibility of dry powders, although further research is needed to clarify the mechanisms behind this enhancement.

Lecithin often needs to be added to these powders to improve dispersibility. PSM treatment improved dispersibility of the powder without the addition of a lecithin, while also increasing the hydrophobicity of the underlying protein. This suggests the protein could either be aggregating in more stable and dispersible structures, or the surface area improvements of PSM overcome the increased hydrophobicity. With the addition of $0.5 \%$ lecithin, PSM was able to improve dispersibility just as efficiently as agglomeration. The further improvement achieved with addition of lecithin could be explained by the ability of PSM to expose more hydrophobic structural pockets, which is a method that has been shown to improve protein lecithin interactions. ${ }^{8}$ Additional investigations on the mechanics behind both of the observed effects are needed in this area.

\section{Conclusions:}


We observed an increase in the solubility and dispersibility of a PSM treated dry powdered whey protein isolate. From this finding it can be concluded that organic materials such as whey protein isolate are candidates for molecular and surface engineering using atmospheric plasma. This validates the use of PSM to enhance both solubility and dispersibility and possibly absorption of substances like dry protein powder used in food production.

\section{Methods:}

This investigation focused on assessing the impact of plasma-surface modification on dry powdered whey protein isolate. Proprietary PSM technology (Ingredient Optimization TM, Plasma Nutrition, USA) was used to effectively mediate the exposure of organic dry powder materials to plasma to enact multiple changes, including peptide level alterations and surface-level alterations. A commercially available dry powder whey protein isolate was obtained as the source material for the study, which also served as the control for the experiment. A portion of the whey protein isolate from this batch was separated and exposed to atmospheric plasma using the PSM technique described above.

Solubility (Methodology based on the International Organization for Standardization (ISO) protocol 8156 "Dried milk and dried milk products -- Determination of insolubility index") and dispersibility (Methodology based on the International Organization for Standardization (ISO) protocol 17758 "Instant dried milk -- Determination of the dispersibility and wettability") analyses were conducted on both a pristine sample of whey protein isolate (control) and a sample of whey protein isolate exposed to atmospheric plasma conditions (experimental) from the same commercially obtained dry whey protein powder batch.

Spectrophotometric analyses were conducted using Eppendorf BioPhotometer Cuvette Spectrophotometer (Hamburg, Germany).

\section{Limitations:}

This study investigated the application of PSM on only one source of dry powdered whey protein isolate, which is common in commercial use. However, there may be variations between the protein powder that was used in this study and other market-available whey protein isolate powders. Therefore, further studies are needed to examine the effect of PSM on a range of dry powder whey protein isolate sources.

\section{Funding Statement:}

This work was financially supported by Plasma Nutrition.

\section{Ethics Statement:}

All experiments were performed in vitro and did not involve the use of any living subject. 
No fraudulence is committed in performing these experiments or during processing of the data. We understand that in the case of fraudulence, the study can be retracted by Matters.

\section{Sources:}

1. Zayas, J.F., 1997. Solubility of proteins. In Functionality of proteins in food(pp. 675). Springer Berlin Heidelberg.

2. Wright, B.J., Zevchak, S.E., Wright, J.M. \& Drake, M.A. (2009). The impact of agglomeration and storage on flavor and flavor stability of whey protein concentrate $80 \%$ and whey protein isolate. Journal of food science, $74(1)$. DOI: 10.1111/j.1750-3841.2008.00975.x

3. Nakai, S. \& Li-Chan, E. (1985). Structure modification and functionality of whey proteins: quantitative structure-activity relationship approach. Journal of Dairy Science, 68(10), pp.2763-2772. DOI: 10.3168/jds.S0022-0302(85)81164-4

4. Wit, J. N. (1989). Functional properties of whey proteins. In: P. F. Fox (Ed.), Developments in dairy chemistry, Vol. 4 (pp. 285-321). London: Elsevier Applied Science.

5. Pelegrine, D.H.G. and Gasparetto, C.A. (2005). Whey proteins solubility as function of temperature and $\mathrm{pH}$. LWT-Food Science and Technology, 38(1), pp.77-80. DOI: 10.1016/j.Iwt.2004.03.013

6. Chu, P.K., Chen, J.Y., Wang, L.P. and Huang, N. (2002). Plasma-surface modification of biomaterials. Materials Science and Engineering: $R$ : Reports, 36(5), pp.143-206. DOI: 10.1016/S0927-796X(02)00004-9

7. Jeong, J.Y., Babayan, S.E., Tu, V.J., Park, J., Henins, I., Hicks, R.F. and Selwyn, G.S. (1998). Etching materials with an atmospheric-pressure plasma jet. Plasma Sources Science and Technology, 7(3), p.282. DOI: 10.1088/0963-0252/7/3/005

8. Henning DR, Baer RJ, Hassan AN \& Dave R. (2006). Major advances in concentrated and dry milk products, cheese, and milk fat based spreads. J Dairy Sci 89:1179-88. DOI: 10.3168/jds.S0022-0302(06)72187-7 